\title{
OTIMIZAÇÃO DO USO DO TRATOR FLORESTAL Forwarder EM FUNÇÃO DA PRODUTIVIDADE, CUSTOS E CAPACIDADE DE CARGA ${ }^{1}$
}

Flávio Cipriano de Assis do Carmo², Nilton Cesar Fiedler ${ }^{3}$, Luciano José Minette e Amaury Paulo de Souza $^{5}$

\begin{abstract}
RESUMO - Esta pesquisa analisou a operação do trator florestal Forwarder em função das variáveis produtividades, custos e potencial de carga visando à sua otimização e consequente redução dos custos de produção. Foi realizada uma análise de sensibilidade para avaliar o grau de variação da produção em relação à redução dos tempos improdutivos. A otimização do uso do compartimento de carga do forwarder foi feita por meio de um estudo de medição do volume de madeira empilhada nas margens das estradas e, em conjunto com o valor da densidade da madeira fornecida pela empresa, da determinação do peso médio (tonelada) de madeira extraída em cada ciclo operacional do forwarder. Os resultados indicaram que, com a eliminação dos tempos improdutivos, é possível aumentar a produção do forwarder e reduzir o custo final da madeira em até $8,24 \%$. Foi verificada uma ineficiência na otimização do potencial de carga do forwarder, de modo que o aumento do compartimento de carga até o limite de peso recomendado pelo fabricante poderá elevar a produtividade da máquina em $32,0 \%$, $44,5 \%$ e 54,7\% para povoamentos com volume médio individual de $0,19 \mathrm{~m}^{3} \cdot \mathrm{arv}^{-1}, 0,15 \mathrm{~m}^{3} \cdot \mathrm{árv}^{-1}$ e $0,10 \mathrm{~m}^{3} \cdot \mathrm{arv}^{-1}$, respectivamente. Foi verificado ainda que, em relação às distâncias médias de extração, não houve diferença significativa na produtividade da máquina até a distância de extração de $200 \mathrm{~m}$.
\end{abstract}

Palavras-chave: Colheita Florestal; Extração florestal; Análise de rendimento.

\section{OPTIMIZATION OF THE USE OF FORESTRY Forwarder BASED ON PRODUCTIVITY, COSTS AND LOAD CAPACITY}

\begin{abstract}
This research examined the operation of forestry tractor Forwarder based on the variables productivity, costs and potential for load aiming its optimization and consequent reduction in production costs. A sensitivity analysis was performed to assess the degree of production variation in relation to the reduction of unproductive times. The optimization of the use of loading compartment of the forwarder was done by means of a measurement study of the volume of wood stacked on the edges of roads and, together with the value of the wood density provided by the company, the determination of average weight (ton) of wood extracted in each operating cycle of the forwarder. The results indicated that, with the elimination of unproductive times it is possible to increase the production of the forwarder and reduce the final cost of wood up to $8.24 \%$. Inefficiency in optimizing the load potential of forwarder was verified, so that the increase of the load compartment until the weight limit recommended by the manufacturer may increase the machine productivity in $32.0 \%$, 44.5\% and $54.7 \%$ for stands with an individual average volume of $0.19 \mathrm{~m}^{3}$. tree $^{-1}, 0.15 \mathrm{~m}^{3}$. tree $^{-1}$ and $0.10 \mathrm{~m}^{3}$.tre $\mathrm{e}^{-1}$, respectively. It was also found that, in relation to average distances of extraction, there was no significant difference in the productivity of the machine until the distance of extraction of $200 \mathrm{~m}$.
\end{abstract}

Keywords: Forest Harvesting; Forest Extraction; Analysis of Income.

\footnotetext{
${ }^{1}$ Recebido em 06.10.2014 aceito para publicação em 07.04.2015.

${ }^{2}$ Universidade Federal do Espírito Santo, Centro de Ciências Agrárias, Programa de Pós Graduação em Ciências Florestais, Jerônimo Monteiro, ES, Brasil. E-mail:<flaviocipriano@hotmail.com>.

${ }^{3}$ Universidade Federal do Espírito Santo, Centro de Ciências Agrárias, Departamento de Ciências Florestais e da Madeira, Jerônimo Monteiro, ES - Brasil. E-mail:<nilton.fiedler@ufes.br>.

${ }^{4}$ Universidade Federal de Viçosa, Departamento de Engenharia Elétrica e de Produção, Viçosa, MG - Brasil. E-mail:<minette@ufv.br>.

${ }^{5}$ Universidade Federal de Viçosa, Departamento de Engenharia Florestal, Viçosa, MG - Brasil. E-mail: $<$ amaury@ufv.br $>$.
} 


\section{INTRODUÇÃO}

Entre as várias etapas do processo produtivo florestal, tem-se a colheita de madeira, que compreende as operações de derrubada, processamento e extração da madeira até as margens das estradas. A colheita florestal é atividade complexa, dado o grande número de variáveis que afetam a produtividade e, consequentemente, os custos operacionais (CANTO et al., 2006). Assim, é de suma importância verificar o melhor sistema de colheita que resulte no menor custo por volume produzido, levando-se em consideração as condições do terreno, a finalidade de uso da madeira e os recursos financeiros disponíveis na execução das atividades.

Segundo Machado e Lopes (2000), o custo de colheita e transporte representa mais da metade do custo final da madeira posta no local de utilização. O conhecimento do custo operacional das máquinas envolvidas no processo produtivo é de suma importância na tomada de decisão, auxiliando, fundamentalmente, o planejamento e controle das operações para a redução dos custos de produção (MACHADO; MALINOVSKI, 1988). Assim, a seleção de máquinas e equipamentos adequados a ser adotada num sistema de colheita florestal constitui um dos grandes desafios para a redução dos custos finais da madeira (BURLA et al., 2012).

O uso de tratores florestais de grande versatilidade e capacidade de carga e o desenvolvimento das operações em três turnos de trabalho, como os forwarders (cuja função é realizar a extração de madeira), propiciam maior disponibilidade das máquinas para a operação, acarretando em menor custo de produção (MACHADO et al., 2008).

Por fim, o planejamento das atividades de colheita de madeira em conjunto com a logística de máquinas e a otimização da sequência de execução do corte dos talhões são etapas de fundamental importância, possibilitando obter menores distâncias de deslocamento entre as unidades de corte e, consequentemente, aumento da produtividade e redução dos custos.

O objetivo desta pesquisa foi realizar uma análise operacional das atividades de extração florestal, visando à otimização do uso do trator florestal Forwarder em razão das variáveis produtividade, custos e potencial de carga para redução dos custos de produção.

Revista Árvore, Viçosa-MG, v.39, n.3, p.561-566, 2015

\section{MATERIAL E MÉTODOS}

\subsection{Características das áreas de estudo}

A pesquisa foi realizada em plantios florestais localizados no Sul do Estado da Bahia. A descrição da área de estudo está representada na Tabela 1.

\subsection{Sistema de colheita de madeira utilizado pela empresa}

O sistema de colheita adotado foi o de toras curtas (cut-to-lenght), sendo as operações de derrubada, processamento e extração da madeira realizadas de forma mecanizada, com o módulo de colheita da empresa composto por 10 harvesters e quatro forwarders. $\mathrm{O}$ harvester (colhedor florestal) executa as atividades de derrubada, descascamento, desgalhamento e destopamento em toras de $6,0 \mathrm{~m}$ de comprimento, com o eito de trabalho constituído por quatro fileiras de árvores e a madeira depositada à esquerda, no sentido de deslocamento da máquina.

O forwarder (trator florestal autocarregável) executa as atividades de extração e empilhamento da madeira na margem das estradas. Para este estudo, foram avaliados forwarders com rodados de pneus $6 \times 6$, peso de 16.800 $\mathrm{kg}$, motor com potência de $111,8 \mathrm{~kW}$, com capacidade de carga de $9.000 \mathrm{~kg}$. Essa máquina trabalhava em três turnos diários de $8 \mathrm{~h}$ ( $24 \mathrm{~h} /$ dia $)$, durante todos os dias da semana.

\subsection{Coleta de dados}

Os dados utilizados nesta pesquisa foram obtidos a partir de dados primários registrados diariamente pela empresa no período produtivo da máquina, de março de 2011 a setembro de 2012. Além disso, realizouse a coleta de dados de campo, por meio de um estudo de tempos e movimentos, utilizando a metodologia proposta por Barnes (1977), caracterizada pelo método de tempos contínuos, dividindo a operação em fases do ciclo de trabalho (deslocamento vazio, carregamento, deslocamento carregado e descarregamento) e realizando análise-piloto com o objetivo de definir o número mínimo de amostras necessárias para um erro de amostragem máximo de $1 \%$. Utilizaram-se cronômetro digital e formulário específico para registro das informações.

Foi realizado o acompanhamento das operações de extração com quatro forwarders in loco, durante o andamento normal das atividades, sem qualquer interferência. 
Tabela 1 - Caracterização da área de estudo. Table 1 - Characterization of the study area.

\begin{tabular}{|c|c|}
\hline \multicolumn{2}{|r|}{ Característica da área de estudo } \\
\hline Municípios abrangidos & $\begin{array}{l}\text { Alcobaça (coordenadas } 17^{\circ} 37^{\prime} 14^{\prime \prime} \mathrm{S} 39^{\circ} 29^{\prime} 29^{\prime \prime} \mathrm{O} \text { ) e Medeiros Neto (coordenadas } \\
\left.17^{\circ} 22^{\prime} 41^{\prime \prime} \mathrm{S} 40^{\circ} 10^{\prime} 10^{\prime \prime} \mathrm{O}\right)\end{array}$ \\
\hline Clima & $\begin{array}{l}\text { Clima tropical úmido, precipitação média anual de } 1.350 \mathrm{~mm} \text {, sendo o período } \\
\text { chuvoso de outubro a dezembro e o período seco de julho a setembro }\end{array}$ \\
\hline Relevo & Plano a suave ondulado com declividade máxima de até $5 \%$ \\
\hline Solo & Varia entre Latossolo Amarelo distrófico e Vermelho-Amarelo distrófico \\
\hline \multicolumn{2}{|r|}{ Característica da espécie de estudo } \\
\hline Espécie & Híbrido das espécies de Eucalyptus grandis e Eucalyptus urophilla \\
\hline Volume médio individual das árvores & 0,$19 ; 0,15$ e $0,10 \mathrm{~m}^{3}$ \\
\hline Volume por hectare & $211 \mathrm{~m}^{3} \cdot \mathrm{ha}^{-1} ; 167 \mathrm{~m}^{3} \cdot \mathrm{ha}^{-1} ; 111 \mathrm{~m}^{3} \cdot \mathrm{ha}^{-1}$ \\
\hline
\end{tabular}

Fonte: INMET, 2013 - Dados da empresa.

\subsection{Análise de sensibilidade em função dos tempos improdutivos}

Para verificar o aumento de produtividade do forwarder, realizou-se uma análise de sensibilidade em razão das variáveis produtividade, custos e potencial de carga. Foi realizada a simulação de quatro cenários relacionados com a redução de tempos improdutivos $(0,25,50$ e $75 \%)$. Esses tempos foram caracterizados pelos tempos em que as máquinas estão disponíveis para operação, mas que não estão sendo utilizadas (por falta de operador, falta de frente de serviço, aguardando carreta prancha e por motivos de fortes chuvas que possam impossibilitar a operação), ou tempo ocioso consumido durante a atividade de manutenção (como aguardando peças e mecânicos).

O primeiro cenário representou a produtividade efetiva das máquinas, o segundo correspondeu à retirada de $25 \%$ dos tempos classificados como improdutivos e a adição destes aos tempos produtivos, e assim sucessivamente até a redução de $75 \%$ dos tempos improdutivos (quarto cenário). Neste estudo, não foi considerado o cenário $100 \%$ devido à grande dificuldade das empresas em trabalhar no ótimo operacional (ausência de gargalos operacionais) e à dependência das condições climáticas que, às vezes, impossibilita a operação das máquinas (a exemplo da ocorrência de chuvas que afetam a capacidade de sustentação e tração das máquinas).

Para a simulação do cenário referente à redução dos custos finais do forwarder em função da diminuição dos tempos improdutivos, foram adotados valores encontrados em trabalhos elaborados por Minette et al. (2004), estimados pela metodologia proposta pela FAO e por Machado e Malinovski (1988). Os custos foram divididos em fixos (depreciação, seguros, juros, impostos) e variáveis (mão de obra, administração, combustível, óleo hidráulico, óleo lubrificante do motor, manutenção e reparos), conforme a metodologia adotada por Minette et al. (2004). Esses autores encontraram valor médio de extração de US $\$ 1,92 . \mathrm{m}^{-3}$, o equivalente a $\mathrm{R} \$ 3,92 \cdot \mathrm{m}^{-3}$ (para a cotação do dólar de US\$1,00, equivalente a $\mathrm{R} \$ 2,03)$.

Tomando por base os valores encontrados por Minette et al. (2004), realizaram-se simulações no sentido de verificar a redução de custos das máquinas para os quatro cenários de produtividade. Assim, os custos fixos permaneceram inalterados, e os custos variáveis foram alterados em razão dos diferentes cenários analisados na empresa.

\subsection{Maximização da utilização das máquinas}

Para obter o maior rendimento na utilização do compartimento de carga dos forwarders, foi realizado um estudo de medição de volumes de madeira empilhada nas margens das estradas e, juntamente com o valor da densidade da madeira no talhão e o número de ciclos consumidos na formação das pilhas, determinou-se o peso médio, em tonelada, da madeira extraída em cada ciclo operacional das máquinas.

Assim, com os dados técnicos de suporte da capacidade de carga das máquinas especificados pelos fabricantes e o peso médio da madeira extraído em diferentes classes de produtividade dos talhões $(0,10$; 0,$15 ;$ e $0,19 \mathrm{~m}^{3}$ por árvore), verificou-se quanto de volume

Revista Árvore, Viçosa-MG, v.39, n.3, p.561-566, 2015 
pode ser acrescentado ao compartimento de carga até atingir o limite de peso estabelecido pelo fabricante.

\subsection{Análise estatística}

Os resultados referentes às fases operacionais de cada turno e módulos de trabalho foram analisados considerando como delineamento inteiramente casualizado. Os dados foram processados por meio de uma análise de variância (ANOVA a 99\% de probabilidade) e, quando significativos, as médias, comparadas pelo teste de Tukey a 99\% de probabilidade.

\section{RESULTADOS}

\subsection{Análise de sensibilidade em razão da diminuição dos tempos improdutivos}

Os resultados da análise de sensibilidade da influência do aumento da produção e redução dos custos em razão da diminuição dos tempos improdutivos são apresentados na Tabela 2 .

\subsection{Maximização da utilização das máquinas}

Após a análise dos indicadores de rendimento, verificou-se a produtividade do forwarder em relação às diferentes distâncias médias de extração. Além disso, foi observada a eficiência da utilização da potência das máquinas (Tabela 3 ).

\section{DISCUSSÕES}

Como pode ser visto, simulando os diversos cenários de redução dos tempos improdutivos, percebeu-se perda de produção média de $10,14 \mathrm{~m}^{3} \cdot \mathrm{h}^{-1}$ (hora efetiva de trabalho) do forwarder, equivalente a uma perda de $19,27 \%$ em relação à produtividade real da máquina.

Foi verificado que os tempos improdutivos contribuíram para a perda da produtividade das máquinas e, consequentemente, para a elevação do custo final da madeira. Entre os tempos improdutivos, o evento mais impactante foi a falta de frente de trabalho (média de $41,83 \%$ do tempo improdutivo da máquina). Isso pode ser explicado pelo desbalanceamento de máquinas nos módulos, ou seja, esses módulos trabalham com um número de forwarders com produtividade final superior ao número de harvesters, proporcionando falta de madeira para extração. Considerando a redução do tempo improdutivo em até $75 \%$, verificou-se que a economia poderia atingir cerca de $8 \%$ do custo final para o forwarder.

Como pode ser observado na Tabela 3, para um povoamento florestal de eucalipto com produtividade de $0,19 \mathrm{~m}^{3}$ por árvore, houve diferença signigicativa a $99 \%$ de probabilidade, pelo teste de Tukey, entre as distâncias médias de extração de 0 a 50 m em relação à última classe de distância de 201 a 250 m, ou seja, até 200 m de extração não há diferença significativa de produtividade da máquina. Esse resultado revela a importância de um planejamento adequado dos talhões, de modo que as rotas de extração da madeira não sejam superiores a $200 \mathrm{~m}$. Além disso, foi observado que, à medida que aumentou a produtividade da floresta em termos de volume individual, aumentou-se a capacidade de otimização

Tabela 2 - Análise de sensibilidade da influência dos tempos improdutivos no aumento da produção e custo final da madeira. Table 2 - Sensitivity analysis of the influence of unproductive times in the increase of the production and final cost of the wood.

\begin{tabular}{lccccccccc}
\hline \multicolumn{1}{c}{ Forwarder } \\
\hline Ano & $\begin{array}{c}\text { Simul } \\
(\%)\end{array}$ & $\mathrm{m}^{3} . \mathrm{hr}^{-1}$ & $\mathrm{R} \$ . \mathrm{m}^{-3}$ & $\begin{array}{c}\mathrm{C} . \mathrm{T} . \\
\left(\mathrm{R} \$ . \mathrm{h}^{-1}\right)\end{array}$ & $\begin{array}{c}\text { C.V. } \\
\left(\mathrm{R} \$ . \mathrm{h}^{-1}\right)\end{array}$ & $\begin{array}{c}\text { C.F. } \\
\left(\mathrm{R} \$ . \mathrm{h}^{-1}\right)\end{array}$ & $\begin{array}{c}\text { C.T.S. } \\
\left(\mathrm{R} \$ . \mathrm{h}^{-1}\right)\end{array}$ & $\begin{array}{c}\text { C.S. } \\
\left(\mathrm{R} \$ . \mathrm{m}^{-3}\right)\end{array}$ & $\begin{array}{c}\text { Red } \\
(\%)\end{array}$ \\
\cline { 2 - 10 } 2010 & 0 & 52,11 & 3,92 & 204,27 & 104,20 & 100,07 & 204,27 & 3,92 & 0,00 \\
& 25 & 55,64 & - & 218,11 & 111,26 & 100,07 & 211,33 & 3,80 & 3,11 \\
& 50 & 59,17 & - & 231,95 & 118,32 & 100,07 & 218,39 & 3,69 & 5,85 \\
& 75 & 62,70 & - & 245,78 & 125,37 & 100,07 & 225,44 & 3,60 & 8,27 \\
2011 & 0 & 53,21 & 3,92 & 208,58 & 106,40 & 102,18 & 208,58 & 3,92 & 0,00 \\
& 25 & 56,44 & - & 221,24 & 112,86 & 102,18 & 215,04 & 3,81 & 2,80 \\
& 50 & 59,66 & - & 233,87 & 119,30 & 102,18 & 221,48 & 3,71 & 5,30 \\
& 75 & 62,89 & - & 246,53 & 125,75 & 102,18 & 227,93 & 3,62 & 7,54 \\
\hline
\end{tabular}

Nota: Simul: Simulação; C.T.: Custo Total; C.V.: Custo Variável (51,01\% para forwarder); C.F.: Somatório de custos fixos, mão de obra e administrativos (48,99\% para forwarder - exceto valores simulados); C.T.S.: Custo Total Simulado (C.V+C.F.); C.S.: Custo de produção simulado; Red.: Redução de custo. Cenário 0: Considerado o cenário testemunha.

Revista Árvore, Viçosa-MG, v.39, n.3, p.561-566, 2015 
Tabela 3 - Produtividade do forwarder em relação a diferentes produtividades dos povoamentos e em diferentes distâncias médias de extração.

Table 3 - Productivity of forwarder regarding different productivities of stands and in different average distances of extraction.

\begin{tabular}{lccccccccc}
\hline $\begin{array}{l}\text { Rend. } \\
\left(\mathrm{m}^{3} \cdot \text { arv }^{-1}\right)\end{array}$ & DME & TMC (min) & kg.viag. ${ }^{-1}$ & Dens. $\left(\mathrm{kg} \cdot \mathrm{m}^{-3}\right)$ & $\mathrm{n}^{\mathrm{o}}$ viag. hora & $\mathrm{m}^{3} \cdot \mathrm{hr}^{-1}$ & ACC $\left(\mathrm{m}^{3}\right)$ & $\mathrm{m}^{3} \cdot \mathrm{hr}^{-1}$ & $\%$ \\
\hline 0.19 & $0-50$ & $12: 57 \mathrm{a}$ & 6815,1 & 496,3 & 4,63 & 63,57 & 4,4 & 83,94 & $32 \%$ \\
& $51-100$ & $13: 37 \mathrm{ab}$ & 6815,1 & 496,3 & 4,40 & 60,41 & 4,4 & 79,77 & $32 \%$ \\
& $101-150$ & $14: 35 \mathrm{ab}$ & 6815,1 & 496,3 & 4,11 & 56,43 & 4,4 & 74,51 & $32 \%$ \\
& $151-200$ & $14: 58 \mathrm{ab}$ & 6815,1 & 496,3 & 4,01 & 55,06 & 4,4 & 72,70 & $32 \%$ \\
& $201-250$ & $15: 40 \mathrm{~b}$ & 6815,1 & 496,3 & 3,83 & 52,59 & 4,4 & 69,44 & $32 \%$ \\
0.10 & 150,5 & $14: 01$ & 5643,8 & 446,3 & 4,28 & 58,76 & 7,52 & 90,95 & $54,7 \%$ \\
0.15 & 150,5 & $14: 19$ & 6229,4 & 452,4 & 4,19 & 57,53 & 6,12 & 83,17 & $44,5 \%$ \\
\hline
\end{tabular}

Nota: Rend: Rendimento; DME: Distância Média de Extração; TMC: Tempo Médio do Ciclo de Extração; kg/viag.: Peso da carga por viagem realizada; Dens.: Densidade da madeira; ACC: Aumento da Caixa de Carga do forwarder; e \%: Percentual do aumento do volume $\left(\mathrm{m}^{3} \cdot \mathrm{hr}^{-1}\right)$.

e peso da caixa de carga do forwarder, porém foram encontrados valores abaixo do limite máximo de carga especificada pelo fabricante, que é de $9.000 \mathrm{~kg}$. Isso pode ser devido à baixa densidade aparente da madeira. Desse modo, pode-se elevar a altura dos fueiros da caixa de carga, desde que não comprometa a estabilidade da máquina e, por consequência, a máquina consiga transportar volume maior de madeira por ciclo operacional.

Com esse aumento da caixa de carga, pode-se elevar a produtividade da máquina em até $54,7 \%$ em povoamentos de baixo volume $\left(0,10 \mathrm{~m}^{3}\right.$ por árvore), ou seja, em determinados talhões, pode haver diminuição no número de máquinas nos módulos e, por consequência, redução no custo final da madeira.

\section{CONCLUSÕES}

De acordo com os resultados, conclui-se que:

A eliminação parcial dos tempos improdutivos pode aumentar a capacidade produtiva do forwarder em 19,27\% e reduzir o custo de produção em $8 \%$.

O aumento da capacidade da caixa de carga do forwarder até o limite recomendado pelo fabricante pode otimizar a produtividade da máquina em até $54,7 \%$, nas condições de povoamentos de baixo volume individual.

Não houve diferença signifivativa na produtividade do forwarder na extração de madeira com distância média de até $200 \mathrm{~m}$. Isso mostra a importância de um planejamento adequado dos talhões, de modo que consiga obter leiautes com dimensionamentos ideais de rotas de extração da madeira sem prejudicar a produtividade das máquinas.

\section{REFERÊNCIAS}

BARNES, R.M. Estudo de movimentos e de tempos: Projeto e medida do trabalho. 6.ed. Americana: Edgard Blucher, 1977. 635p.

BURLA, E.R.; FERNANDES, H.C.; MACHADO, C.C.; LEITE, D.M.; BERNARDES, A.M.Avaliação técnica e econômica do harvester, em diferentes condições operacionais. Engenharia na Agricultura, v.20, n.5, p.412-422, 2012.

CANTO. J.L.; MACHADO, C.C.; GONTIJO, F.M.; JACOVINE, L.A.G. Colheita e transporte florestal em propriedades rurais fomentadas no estado do Espírito Santo. Revista Árvore, v.30, n.6, p.989-998, 2006.

INSTITUTO NACIONAL DE METEREOLOGIA INMET.. Disponível em: http://

www.inmet.gov.br/agrometeorologia/ Acesso em 12 de fev. 2013.

MACHADO C.C.; LOPES E.S. Análise da influência do comprimento de toras de eucalipto na produtividade e custo da colheita e transporte florestal. Revista Cerne, v.6, n.2, p.124-129, 2000.

MACHADO, C.C.; MALINOVSKI, J.R. Ciência do trabalho florestal. Viçosa, MG:

Universidade Federal de Viçosa, 1988. 65p.

Revista Árvore, Viçosa-MG, v.39, n.3, p.561-566, 2015 
MACHADO, C.C.; SILVA, E.N.; PEREIRA, R.S. O setor florestal brasileiro. In: MACHADO, C.C. (Ed.) Colheita florestal. 2.ed. Viçosa, MG: Universidade Federal de Viçosa, 2008. p.15-42.
MINETTE, L.J.; MOREIRA, F.M.T.; SOUZA, A.P.; MACHADO, C.C.; SILVA, K.R. Análise técnica e econômica do forwarder em três subsistemas de colheita em florestas de eucalipto. Revista Árvore, v.28, n.1, p.91-297, 2004. 